\title{
PREVALENCIA DE FACTORES DE RIESGO ASOCIADOS A TRASTORNOS DEL COMPORTAMIENTO ALIMENTARIO EN ADOLESCENTES DE UNA INSTITUCIÓN EDUCATIVA EN CALI, COLOMBIA 2005
}

\section{The prevallence of risk factors associated with eating disorder patterns in adolescents from an educational institution in Calli, Colombia, 2005}

Jorge Martín Rodríguez, M.D., M.Sc., D.Sc. *, Francisco Javier Mina, MSP (C)**

Fecha recibido: diciembre 3/07 - Fecha aceptado: julio 16/08

\section{RESUMEN}

Introducción: los trastornos de la conducta alimentaria (TCA) son un tema de interés actual para la investigación clínica, epidemiológica y básica. El objetivo de este estudio es determinar la prevalencia y las percepciones de riesgo asociadas a TCA en mujeres adolescentes de una institución educativa en la ciudad de Cali.

Materiales y métodos: se realizó un estudio observacional tipo transversal con una muestra de adolescentes procedentes de una institución educativa en Cali, Colombia. Se diseñó y validó un cuestionario estructurado para la recolección de la información; se realizó análisis univariado y bivariado empleando intervalos de confianza (IC) al 95\% y un nivel de significancia $\mathrm{p}<0.05$; se diferenciaron conductas a nivel individual, familiar y social.

Resultados: la edad promedio de las estudiantes fue 13 años, el 7,1\% (IC: 95\% 3,5\% - 10,7\%) refirieron vomitar como medida para reducir peso.

* Epidemiología (C). Médico Universidad Nacional de Colombia. Magíster en Epidemiología. Universidad del Valle. Estudiante de Doctorado en Epidemiología. Instituto Nacional de Salud Pública de México. Cuernavaca, México. Correo electrónico: georod@gmail.com

** Psicólogo de la Universidad Cooperativa de Colombia. Magíster en Salud Pública (C). Universidad del Valle. Psicólogo de la Empresa Social Ladera de Cali. Cali, Colombia.
En las conductas identificadas en sus familiares, el 86,9\% (IC: 95\% 82,2\% - 86,9\%) reportó uso de laxantes para adelgazar, mientras en amigos y pares el 15,7\% (IC: 95\% 10,6\% - 20,8\%) y el 12,6\% (IC: 95\% 8,0\% - 17,2\%) percibieron inducción al vómito y empleo de pastillas para adelgazar, respectivamente. La prevalencia de riesgo para los TCA fue 3,5\%, siendo estadísticamente significativa en las mayores de 15 años y en adolescentes de grados superiores a noveno ( $\mathrm{p}<0,05)$.

Discusión: aunque la prevalencia del riesgo encontrada en esta investigación fue baja respecto a otras, se puede atribuir en parte a razones metodológicas, o porque la edad promedio de estas adolescentes era menor a otros estudios.

Palabras clave: riesgo, trastornos conducta alimentaria, adolescentes.

\section{SUMMARY}

Introduction: eating disorders (ED) are topics of current interest for clinical, epidemiological and basic research. This study was aimed at determining the prevalence and perceptions of risk associated with ED in female adolescents from an educational Institution in the city of Cali. 
Materials and methods: an observational, crosssectional study was carried out with a sample of teenagers from an educational institution in Cali, Colombia. A questionnaire was designed and validated for collecting data. Univariate and bivariate analyses were performed using 95\% confidence intervals and a $\mathrm{P}<0.05$ significance level. Individual, family and social behavior was reported.

Results: students' average age was 13; 7.1\% of them (3.5\%-10.7\% 95\%CI) cited vomiting as a way of losing weight. Their relatives identified $86.9 \%$ $(82.2 \%-86.9 \% 95 \% \mathrm{CI})$ of them using laxatives to lose weight while friends and peers reported $15.7 \%(10.6 \%-20.8 \% 95 \% \mathrm{CI})$ inducing vomiting and seeing $12.6 \%(8.0 \%-17.2 \% 95 \% \mathrm{CI})$ of them using diet pills. There was 3.5\% prevalence of risk of $\mathrm{ED}$, this being statistically significant in those aged more than 15 and in girls from the ninth grade and above $(\mathrm{P}<0.05)$.

Discussion: although the prevalence of risk found in this research was low in relation to others, it can be partly attributed to methodological reasons or these adolescents' average age, as they were younger than in other studies.

Keys words: risk, eating disorder, teenager.

\section{INTRODUCCIÓN}

En las últimas décadas los trastornos de la conducta alimentaria (TCA) han sido tema de interés para la investigación clínica, epidemiológica y de las ciencias básicas. Es un problema que ha afectado principalmente a un sector de la población, las mujeres jóvenes. Los pacientes con TCA presentan características que afectan los tres sistemas de respuesta (cognitivo, fisiológico y motor), así como diferentes problemas asociados a su diagnóstico básico han requerido atención y manejo especializado. ${ }^{1}$ La alarma creada con los $\mathrm{TCA}^{2}$ contrasta con el escaso desarrollo de estudios epidemiológicos y la evaluación de intervenciones preventivas. ${ }^{3}$

Para la Asociación Americana de Psiquiatría, los TCA son trastornos caracterizados por severos disturbios en el comportamiento alimentario con altos niveles de sintomatología obsesiva-compulsiva, acompañados de alteraciones en la percepción de la imagen y peso corporal. Los TCA principalmente descritos son la anorexia nerviosa, la bulimia y los trastornos del comportamiento alimentario inespecífico. $^{4}$

La anorexia nerviosa es un trastorno biopsicosocial de carácter compulsivo en un(a) paciente generalmente retraído(a), rígido(a), auto devaluativo(a), sobreprotegido(a) y preocupado(a) en exceso por su peso, con marcado interés por comidas dietéticas, ejercicio y disminución de relaciones sociales que involucran comidas y bebidas. Se mantienen en un peso debajo de lo mínimo normal (15\% abajo de lo esperado según tablas estandarizadas para edad y talla, o un índice de masa corporal igual o inferior a $\left.17,5 \mathrm{~kg} / \mathrm{m}^{2}\right)$, con ausencia de al menos tres ciclos menstruales consecutivos. La disminución de peso se logra a partir de dietas inicialmente selectivas, luego generalizadas, acompañadas de ejercicio intenso y empleo de laxantes y/o diuréticos. ${ }^{4,5}$

El paciente puede manifestar síntomas depresivos e incluso criterios para trastorno depresivo mayor, siendo en algunas ocasiones éste el factor desencadenante del TCA. Entre sus complicaciones se han descrito: retraso de la madurez sexual, del crecimiento y del desarrollo físico, bradicardia, hipotensión ortostática, osteopenia irreversible, desequilibrio hidroelectrolítico, trastornos de la movilidad gastrointestinal, infertilidad y cambios estructurales en el cerebro, que incluyen disminución de las sustancias blanca y gris, generando deterioro cognitivo durante y después de la enfermedad. ${ }^{4,6,7}$

En cuanto a la bulimia, es un TCA de tipo compulsivo obsesivo consistente en episodios de ingesta masiva de alimentos con la sensación de pérdida del control; se inicia como un cuadro caracterizado por crisis bulímicas o atracones (rápido consumo de alimentos en gran cantidad, de alto contenido calórico) en menos de 2 horas de duración y sensación de pérdida de autocontrol por más de dos veces por semana durante más de tres meses, asociado a dolor abdominal intenso y vómito inducido posteriormente 
(80-90\% de los casos), que permite la disminución del malestar por plenitud y temor de ganancia de peso. ${ }^{5}$ La paciente suele utilizar adicionalmente laxantes, diuréticos, dieta y ejercicios vigorosos; la persona con bulimia se avergüenza de su problema e intenta ocultar los síntomas. Los episodios bulímicos pueden ser planeados o no; generalmente ocurren luego de estados de ánimo alterados: disfóricos vs. estresores con intensa sensación de hambre y cesan cuando el individuo siente malestar o sensación de plenitud luego de la ingesta. El peso de estos individuos es generalmente normal o ligeramente arriba o abajo de lo normal.

Entre las complicaciones de la bulimia se encuentran: desequilibrio hidroelectrolítico, sangrado digestivo alto, erosión del esmalte dental, agrandamiento de las parótidas, hiperamilasemia, cardiomiopatías; anormalidades del intestino grueso debidas al abuso de laxantes, bradicardia, hipotensión, déficit del eje hipotálamo-hipófisis-tiroides, dolor precordial, edema, cambios en la piel y el cabello y muerte súbita. ${ }^{4,5}$

Respecto a los TCA no especificados, se caracterizan por cuadros clínicos que no cumplen los criterios para ningún trastorno de la conducta alimentaria específica, algunos ejemplos son: mujeres con criterios diagnósticos para anorexia, pero con menstruaciones regulares, o que tengan índices de masa corporal normales para la edad o que cumplan criterios para bulimia, pero con una frecuencia menor de atracones (una vez por semana, por sólo un mes); empleo de conductas inapropiadas luego de ingerir pequeñas cantidades de comida en individuos con peso normal (provocación del vómito luego de ingerir alimentos); masticar o expulsar sin tragar cantidades importantes de comida; atracones con ausencia de conductas compensatorias inapropiadas. ${ }^{4,8,9}$

La prevalencia de estos eventos ha sido mayor en clases sociales altas y medias; no obstante, en los últimos años su distribución tiende a homogeneizarse. Es conocida también la asociación con determinadas profesiones (gimnastas, bailarinas), donde se observan a menudo casos subclínicos o atípicos. ${ }^{10}$
Las diferentes líneas de investigación sobre los posibles factores etiopatogénicos de los TCA llevan a la consideración de un origen multicausal; se han estudiado factores socioculturales, familiares, neuropsíquicos, endocrinos e incluso genéticos asociados a su ocurrencia. ${ }^{11,12}$ Estudios longitudinales indican que cinco años después del diagnóstico $25 \%$ de los pacientes siguen padeciendo anorexia, $40 \%$ presentan síntomas depresivos y 25\% síntomas obsesivos. ${ }^{13,14}$

Los TCA son un padecimiento que está afectando con más frecuencia a personas en edades tempranas, las adolescentes empiezan a presentar comportamientos como mirarse al espejo, pesarse, competir por disminuir su frecuencia de consumo de alimentos $;{ }^{15,16}$ por este motivo se seleccionó una institución educativa media - IEM de la ciudad de Cali, en la cual se tenían indicios de conductas de riesgo para este tipo de eventos (además históricamente, dentro del contexto colombiano, se sabe que en la ciudad de Cali los medios de comunicación resaltan la figura y belleza femenina), con el objetivo de determinar la prevalencia y las percepciones de riesgo asociadas a TCA en mujeres adolescentes de una institución educativa en la ciudad de Cali.

\section{MATERIALES Y MÉTODOS}

Se realizó un estudio observacional descriptivo, tipo encuesta transversal, ${ }^{17,18}$ la población objeto del estudio fueron preadolescentes y adolescentes entre 10 y 18 años matriculadas en una institución de IEM de la ciudad de Cali, Valle del Cauca, Colombia.

Por un muestreo no probabilístico se incluyeron 198 estudiantes de sexo femenino, esto correspondió a una tasa de respuesta del 90\% de las estudiantes matriculadas en los diferentes grados escolares de la IEM.

Los datos se recolectaron por medio de un cuestionario estructurado ${ }^{19}$, el cual había sido previamente validado mediante una prueba piloto en una institución educativa homóloga de la ciudad de Cali, con grupos de adolescentes entre 12 y 16 años; esta prueba permitió ajustar y estandarizar 
la sintaxis, redacción y contenidos del cuestionario para su uso posterior en la IEM seleccionada.

El cuestionario comprendía: información sociodemográfica, comportamiento alimentario autoreferido por las entrevistadas y conductas alimentarias observadas por ellas en sus familiares (padres, hermanos, primos) y amigos (pares o compañeros del colegio); esta información fue manejada en los resultados como dimensiones de nivel individual, familiar y social.

Además se exploraron aspectos relacionados con los factores de riesgo reportados por la literatura: percepción de la figura corporal por sí mismo, por los padres y compañeros, relaciones personales con los padres y amigos, nivel de resolución de conflictos, desempeño escolar y prácticas para reducir peso.

Los datos fueron recopilados durante el mes de noviembre de 2005, luego de dos visitas a la IEM, por un equipo conformado por un coordinador de campo, dos profesionales supervisores y cinco encuestadores, quienes atendían las inquietudes de las adolescentes ante alguna duda presentada durante el desarrollo de la encuesta. El equipo de profesionales y los encuestadores participaron en el proceso desde la validación del instrumento en la prueba piloto, de tal forma que éste era conocido por todos en el momento de su aplicación final; de esta forma se estandarizaron y homogeneizaron las técnicas de recolección de la información. ${ }^{20}$

Para la captura y verificación de entrada de la información se creó una máscara de captura por medio del paquete estadístico Epiinfo 6,04, ${ }^{21}$ para el procesamiento, limpieza y análisis de la información se empleó Statistical Package for the Social Sciences-SPSS, versión 12,0. ${ }^{22}$ En el análisis se calcularon proporciones medias e intervalos de confianza al 95\%; la prevalencia de riesgo de padecer TCA se calculó con base a los criterios empleados por Unikel y colaboradores, fijando un punto de corte en siete dentro de la escala manejada por estos autores. ${ }^{19}$
Los resultados de las dimensiones (individual, familiar y social) se organizaron para cada variable con el porcentaje de respuestas afirmativas; se empleó un nivel de significancia descriptivo de 0,05 para comparar diferencias entre grupos de edad y años de escolaridad; los intervalos de confianza al 95\% se usaron para comparar los comportamientos autorreferidos por las adolescentes (nivel individual) y los observados en sus padres, hermanos y primos (nivel familiar) y en amigos, pares y compañeros (nivel social).

Este trabajo se rigió por las pautas y principios éticos planteados en la declaración de Helsinki ${ }^{23}$ y en la resolución 8430 de 1993 del antiguo Ministerio de Salud de Colombia, ${ }^{24}$ contó con la aprobación del comité de ética de la Fundación Universitaria San Martín, sede Cali. Se garantizó el anonimato y la confidencialidad de la información suministrada por los entrevistados, mediante el diseño e implementación de un consentimiento informado escrito, el cual fue aceptado por las directivas de la institución educativa, además fue aprobado por cada grupo de estudiantes, previo a la aplicación de la encuesta.

\section{RESULTADOS}

Las 198 adolescentes entrevistadas eran estudiantes de 6 a 11 grado y tenían entre 10 a 18 años, con una edad promedio de 13 años, el 75\% de las estudiantes tenían 15 o menos años; esta distribución se observa en la tabla 1.

Con respecto a las conductas y prácticas relacionadas con los TCA, en la tabla 2 se observan la distribución y frecuencia con respuestas afirmativas de estos hallazgos.

En la dimensión individual se resalta la preocupación por engordar y quienes consideran que han comido demasiado; sólo 2,5\% de las entrevistadas reportaron el empleo de agua y laxantes para adelgazar; no obstante 5,6\% reportaron uso de pastillas para adelgazar y 7,1\% refirieron vomitar como medida para reducir de peso.

Respecto a la dimensión familiar, la principal práctica observada por las adolescentes en sus 


\begin{tabular}{|l|c|c|c|c|c|}
\hline \multicolumn{7}{|c|}{ Tabla 1. Distribución de edad y grados escolares entre adolescentes } \\
de una institución educativa, Cali - 2005.
\end{tabular}

\begin{tabular}{|c|c|c|c|c|c|c|c|c|c|}
\hline \multirow{3}{*}{\begin{tabular}{|l}
\multicolumn{1}{|c}{ Dimensión } \\
Variable \\
Preocupación por engordar \\
\end{tabular}} & \multicolumn{3}{|c|}{ Individual } & \multicolumn{3}{|c|}{ Familiar } & \multicolumn{3}{|c|}{ Social } \\
\hline & \multirow{2}{*}{$\begin{array}{c}\% \\
76,6\end{array}$} & \multicolumn{2}{|c|}{ IC $(\mathrm{LS}-\mathrm{LI}) *$} & \multirow{2}{*}{$\begin{array}{c}\% \\
67,8\end{array}$} & \multicolumn{2}{|c|}{$\mathrm{IC}(\mathrm{LS}-\mathrm{LI}) *$} & \multirow{2}{*}{$\begin{array}{c}\% \\
79,3\end{array}$} & \multicolumn{2}{|c|}{$\mathrm{IC}(\mathrm{LS}-\mathrm{LI}) *$} \\
\hline & & 82,5 & 70,7 & & 74,3 & 61,3 & & 84,9 & 73,7 \\
\hline Han comido demasiado & 53,1 & 60,1 & 46,1 & 24,2 & 30,2 & 18,2 & 32,3 & 38,8 & 25,8 \\
\hline Pérdida del control al comer & 29,9 & 36,3 & 23,5 & 31,3 & 37,8 & 24,8 & 38,1 & 44,9 & 31,3 \\
\hline Vómito después de la comida & 7,1 & 10,7 & 3,5 & 14,1 & 18,9 & 9,3 & 15,7 & 20,8 & 10,6 \\
\hline Ayuno para bajar de peso & 12,2 & 16,8 & 7,6 & 27,9 & 34,2 & 21,6 & 35,0 & 41,7 & 28,3 \\
\hline Dietas para adelgazar & 39,9 & 46,7 & 33,1 & 70,2 & 76,6 & 63,8 & 72,1 & 78,4 & 65,8 \\
\hline Ejercicio para bajar de peso & 31,3 & 37,8 & 24,8 & 80,3 & 85,8 & 74,8 & 75,6 & 81,6 & 69,6 \\
\hline Uso de pastillas para adelgazar & 5,6 & 8,8 & 2,4 & 25,3 & 31,4 & 19,2 & 12,6 & 17,2 & 8,0 \\
\hline Uso de agua para adelgazar & 2,5 & 4,7 & 0,3 & 63,1 & 69,7 & 56,5 & 56,1 & 63,0 & 49,2 \\
\hline Uso de laxantes para adelgazar & 2,5 & 4,7 & 0,3 & 86,9 & 91,6 & 82,2 & 6,1 & 9,4 & 2,8 \\
\hline
\end{tabular}

* Intervalos de confianza al 95\% con LS: límite superior y LI: límite inferior

familiares (padres, hermanos, primos) fue ejercitarse para bajar de peso: 80,3\%, (IC 95\% $74,8 \%$ - 85,8\%), práctica superior respecto al nivel individual: 31,3\% (IC 95\% 24,8\% - 37,8\%); aunque al 67,8\% (IC 95\% 61,3\% - 74,3\%) de los familiares les preocupa engordar, no fue diferente a lo autorreportado por las adolescentes: 76,6\% (IC 95\% 70,7\% - 82,5\%); también es de resaltar que, aunque $86,9 \%$ de las entrevistadas reportó que sus familiares usan laxantes para adelgazar, ésta no fue una práctica común entre las adolescentes; igualmente, aunque ellas observan que uno de cada cuatro familiares empleaban pastillas para reducir de peso, ésta tampoco fue una práctica que se haya implementado a nivel individual.

Respecto a la dimensión social, las adolescentes observan que a sus amigos, pares y compañeros en un 79,3\% les preocupa engordar (IC 95\% $73,7 \%$ - 84,9\%), siendo esta proporción similar a lo encontrado a nivel individual 76,6\% (IC 95\% $70,7 \%-82,5 \%)$; para controlar esto, refirieron que $75,6 \%$ realizan ejercicio y 72,1\% dieta. Inducción de emesis la observaron en 15,7\%, siendo superior a las prácticas hechas a nivel individual, tal como se reportó previamente; algo similar ocurre con el empleo de pastillas, observada en 12,6\% de los 
amigos, pero tan sólo en el 5,6\% a nivel de las entrevistadas.

En la tabla 3 se observan las percepciones reportadas por las adolescentes respecto a los comentarios realizados por sus familiares y amigos, en cuanto a situaciones de riesgo asociados a los TCA. Para resaltar, las entrevistadas refirieron que 79,8\% de sus familiares y $77,7 \%$ de sus amigos les realizaban comentarios negativos en relación a su apariencia física; 29,4\% se sentían inconformes con su figura física; $31,3 \%$ y $28,8 \%$ habían recibido sugerencias familiares para bajar de peso o adelgazar. Una de cada cuatro estudiantes, manifestó estar convencida de que la figura física es relacionada con el éxito.

\begin{tabular}{|c|c|c|c|c|c|}
\hline Variable & Frecuencia & Resultado & $\%$ & IC $(1$ & LI)* \\
\hline \multirow{2}{*}{ Comentarios familia apariencia física } & \multirow{2}{*}{198} & Positivos & 20,7 & 26,3 & 15,1 \\
\hline & & Negativos & 79,8 & 84,9 & 73,7 \\
\hline \multirow{2}{*}{ Relaciones con los padres } & \multirow{2}{*}{198} & Buenas & 80,2 & 85,7 & 74,7 \\
\hline & & Deficientes & 19,2 & 24,7 & 13,7 \\
\hline \multirow{2}{*}{ Conflictos con la familia } & \multirow{2}{*}{198} & Ausencia & 67,2 & 73,7 & 60,7 \\
\hline & & Presencia & 32,8 & 39,3 & 26,3 \\
\hline \multirow{2}{*}{ Forma de resolver conflictos con la familia } & \multirow{2}{*}{67} & Fácilmente & 37,3 & 48,9 & 25,7 \\
\hline & & Difícilmente & 62,7 & 74,3 & 51,1 \\
\hline \multirow{2}{*}{ Comentarios amigos apariencia física } & \multirow{2}{*}{197} & Positivos & 22,3 & 28,1 & 16,5 \\
\hline & & Negativos & 77,7 & 83,5 & 71,9 \\
\hline \multirow{2}{*}{$\begin{array}{l}\text { Comentarios amigos y familia peso } \\
\text { corporal }\end{array}$} & \multirow{2}{*}{196} & Bien & 56,1 & 63,0 & 49,2 \\
\hline & & Bajo o Alto & 43,9 & 50,9 & 36,9 \\
\hline \multirow{2}{*}{ Relaciones con los amigos } & \multirow{2}{*}{197} & Buenas & 77,2 & 83,1 & 71,3 \\
\hline & & Deficientes & 22,8 & 28,6 & 17,0 \\
\hline \multirow{2}{*}{ Forma de resolver conflictos con amigos } & \multirow{2}{*}{44} & Fácilmente & 52,3 & 67,1 & 37,5 \\
\hline & & Difícilmente & 47,7 & 62,5 & 32,9 \\
\hline \multirow{2}{*}{ Desempeño escolar último año } & \multirow{2}{*}{197} & Bueno & 81,7 & 87,1 & 76,3 \\
\hline & & Regular & 18,3 & 23,7 & 12,9 \\
\hline \multirow{2}{*}{ Percepción apariencia física } & \multirow{2}{*}{197} & Conforme & 70,6 & 77,0 & 64,2 \\
\hline & & Inconforme & 29,4 & 35,8 & 23,0 \\
\hline \multirow{2}{*}{ Figura física y éxito } & \multirow{2}{*}{198} & Casi siempre & 25,4 & 31,5 & 19,3 \\
\hline & & Algunas veces & 74,6 & 80,7 & 68,5 \\
\hline \multirow{2}{*}{ Sugerencias familia cambio de peso } & \multirow{2}{*}{198} & Mantener peso & 68,7 & 75,2 & 62,2 \\
\hline & & Adelgazar & 31,3 & 37,8 & 24,8 \\
\hline \multirow{2}{*}{ Sugerencias amigos cambio de peso } & \multirow{2}{*}{198} & Mantener peso & 71,2 & 77,5 & 64,9 \\
\hline & & Adelgazar & 28,8 & 1,8 & 1,3 \\
\hline \multirow{2}{*}{ Recomendaciones familia para adelgazar } & \multirow{2}{*}{56} & Mantener peso & 71,2 & 2,5 & 1,6 \\
\hline & & Adelgazar & 28,8 & 2,5 & 1,6 \\
\hline
\end{tabular}

* Intervalos de confianza al 95\% con LS: límite superior y LI: límite inferior 
La prevalencia de los TCA para este grupo de adolescentes, con base a los criterios de Unikel y colaboradores ${ }^{19}$ fue de 3,5\% en las adolescentes. Empleando los mismos criterios, la prevalencia de riesgo que observaron las adolescentes en sus familiares fue $26,3 \%$ y en sus amigos fue 22,2\%. La prevalencia de este riesgo fue estadísticamente significativa en las adolescentes mayores de 15 años de edad $(\mathrm{P}<0,01)$ y en las adolescentes de grados superiores a noveno $(\mathrm{P}<0,05)$.

\section{DISCUSIÓN}

Los TCA son un tema de actualidad en el sector de la salud y en la población en general, han tenido en los últimos años un gran impacto publicitario, involucrando diversos medios de comunicación. Las repecursiones que experimentan las personas con TCA involucran varios aspectos: personalidad, sexualidad, socialización; estabilidad emocional, propia y familiar, y a los sistemas de seguridad social. $^{2,5-8,25,26}$

Rara vez aparecen de forma aislada, se asocian usualmente a otras patologías. Braun, et al. ${ }^{27}$ utilizando criterios del DSM-III-R ${ }^{28}$ en 105 pacientes con TCA, encontraron que $82 \%$ tenían, además de TCA, otros diagnósticos como: trastornos de ansiedad, depresión, alteraciones de la personalidad y abuso de sustancias (principalmente medicamentos inhibidores del apetito y ansiolíticos). Las pacientes anoréxicas desarrollaban primero el TCA y posteriormente otra alteración psicológica.

En la presente investigación, el ayuno, la dieta y el ejercicio se encuentran como las formas predilectas desarrolladas por las adolescentes para bajar de peso; algo similar ocurre en las adolescentes norteamericanas, un $60 \%$ anhelan reducir peso. ${ }^{29}$ Estas conductas también las perciben en sus familias o pares, con el agravante de que se observa en proporciones altas el empleo de pastillas $(25,3 \%)$ y laxantes $(86,3 \%)$ para bajar de peso. Esta situación de aprendizaje por imitación y modelamiento ${ }^{30}$ puede estar generando que 5,6\% de las encuestadas, estén usando pastillas para adelgazar y 7,1\% vomiten como medida para reducir de peso. Las conductas de TCA observadas en las familias y en amigos se pueden comportar como factores de riesgo directo.

Estos resultados son similares a los hallazgos de una investigación realizada en estudiantes en Medellín; en una muestra no representativa de 972 adolescentes de secundaria, se evidenció una alta frecuencia de ideas, sentimientos y conductas de riesgo para TCA: al 77\% de las alumnas les aterraba la idea de ganar peso, 41\% tenía atracones, 33\% se sentía culpable después de comer, 16\% sentía que la comida controlaba su vida y $8 \%$ se inducía el vómito. ${ }^{31}$ Hallazgos similares se encontraron en una investigación realizada por la Universidad Nacional, donde se determinó que los TCA se podían convertir en un problema de salud pública. ${ }^{12}$

La prevalencia de riesgo para los TCA en este grupo de adolescentes fue del 3,5\%; empleando los mismos criterios, las estudiantes perciben en sus familias un 26,3\% de riesgo y en sus amigos 22,2\% de riesgo para TCA; esto es una oportunidad para enfocar el trabajo de prevención primaria involucrando a padres, familiares y/o personas del ámbito no académico (por ejemplo sociedad de padres de familia, círculos de amigos, entre otros). Esta cifra es mayor a la reportada por la Asociación Americana de Psiquiatría, ${ }^{28}$ no obstante, allí se reportan prevalencias del evento, mientras en esta investigación se estima una prevalencia del riesgo usando los criterios mencionados. ${ }^{19}$ Estos resultados son menores a los evidenciados en un grupo de mujeres jóvenes de Venezuela, ${ }^{32}$ quienes reportan un riesgo de 8,8\%; aunque estas diferencias pueden ser por razones metodológicas o porque la edad promedio de las mujeres en esa investigación era significativamente diferente a las entrevistadas en Venezuela $(\mathrm{P}<0,05)$.

Los TCA afectan más a mujeres que a hombres, ${ }^{5,12,19}$ esta investigación tuvo la limitante de no determinar esta diferencia, teniendo en cuenta, desde el diseño, que las seleccionadas eran mujeres adolescentes entre 10 y 18 años. En este estudio, 
al igual que lo reportado por otros autores, la autopercepción acerca de la apariencia física y los comentarios negativos acerca del peso corporal por parte de los pares, pueden ser factores de riesgo para generar TCA. 6,13,18,20

Otros investigadores han estimado la prevalencia de la anorexia nerviosa entre el $0,5-1 \%{ }^{5,8,25,26}$ con una mortalidad aproximada del 10\% de los casos. ${ }^{33}$ Sin embargo, existe controversia en la variación, en los últimos quince años, de la prevalencia de los TCA, algunos investigadores reportan que el número de pacientes remitidos a especialistas ha sido constante $;^{34}$ no obstante, otros han observado incremento de las consultas por TCA. ${ }^{31}$

El problema afecta más a las mujeres con una razón de 10 a 1 respecto a los hombres, con una prevalencia en jóvenes adultas del 1-3\%. Investigadores españoles han estimado prevalencias de TCA del 4\% al 5\% en mujeres escolarizadas de 12 a 21 años. En varones han sido escasos los estudios, la prevalencia, aunque menor, puede llegar al 0,9\%. ${ }^{11}$ En Zaragoza el 16,3\% de las jóvenes de secundaria se situó por encima del punto de corte y el 4,5\% cumplía criterios diagnósticos de anorexia o bulimia. ${ }^{35}$ En Colombia, el estudio de salud mental en una muestra de 15.045 entrevistados, estimó una prevalencia de TCA a lo largo de la vida de 2,3\%, sin encontrar diferencias por género. ${ }^{36}$

La implementación de un diseño transversal como éste, permite identificar la prevalencia de los factores de riesgo de los TCA en el grupo de adolescentes. Algunos de los hallazgos fueron compatibles con los existentes en la literatura; no obstante, es importante precisar que un diseño como éste, no establece causalidad ni asociación etiológica entre una posible exposición y el evento de interés investigado. ${ }^{18}$ De la misma forma, emplear un muestreo no probabilístico limita la validez externa o inferencia de los resultados a una población más amplia; ${ }^{37,38}$ sin embargo, ante la escasez de estudios de esta temática, ${ }^{3}$ esta investigación es una fuente primaria que alimentará y estimulará el estudio de este problema para el diseño de estrategias de prevención y control.
Los autores consideramos poco probable que se haya presentado sesgo de información o algún error de medición, teniendo en cuenta que los procesos de validación implementados desde el diseño (que incluyó la estandarización del instrumento, la homogeneidad empleada en la recolección, procesamiento y análisis de la información) controlan y previenen los posibles errores sistemáticos de este tipo que se puedan presentar. $^{20,39}$

\section{CONCLUSIONES}

Para la prevención y manejo de los TCA, teniendo en cuenta la actual influencia de los medios de comunicación y de los pares, la imitación que hacen los adolescentes de algunos padres o familiares, además de fomentar la investigación para identificar sus determinantes en diferentes contextos, hace necesario estimular procesos de prevención primaria y actividades educativas entre los integrantes de las comunidades educativas (padres, profesores y otros actores). Para prevenir la ocurrencia de los TCA se recomienda supervisar el estado de salud de las adolescentes, principalmente mayores de 15 años o que se encuentren en los últimos años de secundaria, con el objeto de realizar procesos de detección temprana y remisión para manejo profesional oportuno. ${ }^{8,12,13,22}$

\section{AGRADECIMIENTOS}

Los autores agradecen al personal de la Institución Educativa Nuestra Señora de Fátima de la ciudad de Cali, a las estudiantes de V semestre del II semestre de 2005 y a las directivas de la Facultad de Medicina de la Fundación Universitaria San Martín, sede Cali por el apoyo dado a la presente investigación.

\section{REFERENCIAS}

1. Mateos-Parra A, Solano-Pinto N. Trastornos de la alimentación: anorexia y bulimia nerviosas. Psicología. Com [online]1997;1:1.

2. Bosch X. Please don't pais the paella: eating disorders upset Spain. JAMA 2000;283:1405-10. 
3. Leung F, Geller J, Katzman M. Issues and concerns associated with different risk models for eating disorders. Int J Eat Disord 1996;19:249-56.

4. American Psychiatric Association. Diagnostical and Statistical Manual of Mental Disorders. 4th ed. Washington DC: American Psychiatric Press; 1994.

5. Backer AE, Grinspoon SK, Klibanski A, Herzog DB. Eating disorders. N Engl J Med 1999;340:1092-8.

6. Herpertz-Dahlmann BM, Wewetzer C, Remschmidt H. The predictive value of depression in anorexia nervosa. Acta Psychiatr Scand 1995;91:114-9.

7. Stice E, Shaw H y Marti N. A meta analytic review of eating disorders prevention programs: encouraging findings. Annu Rev Clin Psychol 2007;3:207-31.

8. Aguinaga M, Fernández LJ, Varo JR. Trastornos de la conducta alimentaria. Revisión y actualización: Eating Disorders. Anales del Sistema Sanitario de Navarra 2000;23:279-92.

9. Instituto Nacional de Salud Mental de los Estados Unidos. Desórdenes alimentarios: Hechos acerca de los desórdenes de la alimentación y las alternativas de solución, 2003. Visitado 2007 Feb 25. Disponible en: http://www.nihm.nih.gov/publicat/index.cfm

10. Ash JB, Piazza E, Changing symptomatology in eating disorder. Int J Eat Disord 1995;18:27-38.

11. Ruiz-Lázar PM, Comet-Cortés P. Consideraciones metodológicas acerca de la prevalencia de los trastornos de la conducta alimentaria. Med Clin (Barc) 2000; 115:278-9.

12. Ángel L, Vásquez R, Chavarro K. Prevalencia de trastornos del comportamiento alimentario en estudiantes de la Universidad Nacional de Colombia sede Santafé de Bogotá. Acta Med Col 1997;22:111-9.

13. Toro J. El cuerpo como delito. Anorexia, Bulimia, cultura y sociedad. Barcelona: Ariel Ciencia; 1996.

14. Schmidt U, Treasure J, Anorexia nervosa: valued and visible. A cognitive-interpersonal maintenance model and its implications for research and practice, $\mathrm{Br} \mathrm{J}$ Clin Psychol 2006;45:343-66.

15. Misra M, Tsai P, Anderson EJ, Hubbard JL, Gallagher K, Soyka LA, et al. Nutrient intake in community-dwelling adolescent girls with anorexia nervosa and in healthy adolescents. Am J Clin Nutr 2006;84:698-706.

16. Hill JA. Motivation for eating behaviour in adolescent girls: the body beautiful. Proc Nutr Soc 2006;65:376-84.
17. Roca J, MuñozA. Los estudios de prevalencia. En: Martínez F, Antó JM, Castellanos PL. Salud Pública. Madrid: McGraw Hill Interamericana; 1998. p. 179-98.

18. Hernández B, Velasco E. Encuestas transversales. En: Hernández M. Epidemiología: diseño y análisis de estudios. México: Editorial Médica Panamericana; 2007. p. 191-215.

19. Unikel C, Bohórquez J, Carrero S. Validación de un cuestionario breve para medir conductas alimentarias de riesgo. Salud Pública Méx 2004;46:509-15.

20. Szklo M, Nieto J. Epidemiología intermedia. Conceptos y aplicaciones. Madrid: Ediciones Díaz de Santos; 2003.

21. Epiinfo 6.04. Center for Disease Control and Prevention. Atlanta; 1996.

22. Statistical Package for the Social Sciences - SPSS, Version 12.0, 2004.

23. Asociación Médica Mundial. Declaración de Helsinki, última actualización octubre de 2000.

24. Ministerio de Salud de Colombia. Resolución 8430, 1993. p. 1-4.

25. González A, Kohn MR, Clarke SD. Eating disorders in adolescents. Aust Fam Phys 2007;36:614-9.

26. Hamilton JD. Eating disorders in preadolescent children. Nurse Pract 2007;32:44-8.

27. Braun DL, Sunday SR, Halmi KA. Psychiatric comorbidity in patients with eating disorders. Psychol Med 1994;24:859-67.

28. American Psychiatric Association. Manual Diagnóstico y Estadístico de los Trastornos Mentales. (DSM-III-R). (3 ${ }^{\mathrm{a}}$ Ed. rev.). Versión española. Barcelona: Masson; 1987.

29. Prouty AM, Protinsky HO, Canadi D. Collage women: eating behaviors and held-seeking preferentes. Adolescent 2002;37:353-63.

30. Schunk D. Teorías del Aprendizaje. Segunda Edición. México: Prentice-Hall Panamericana; 1997.

31. Ramírez AL, Moreno S, Yépez MM, Pérez GM, Posada AC, Roldan L, Prevalencia de la Anorexia Bulimia en Adolescentes de Medellín, Grupo Académico de Salud Mental de las Mujeres de la Universidad de Antioquia, 2003. Visitado 2007 Ene 30. Disponible en: http:// www.editorialudea.com/saludtextos.html

32. Lugli Z, Vivas E, Conductas y actitudes sugestivas de riesgo de trastornos del comportamiento alimentario en estudiantes universitarios venezolanos. RFM 2006; 29:168-73. 
33. Silber T. Anorexia nervosa, morbidity and mortality. Pediatr Ann 1984;13:851-9.

34. Fairburn CG, Hay PJ, Welch SL. Binge eating and bulimia nervosa: distribution and determinants. En: Fairburn C, Wilson T (eds). Binge eating: nature, assessment and treatment. New York: Guilford Press; 1993.

35. Ruiz P, Alonso JP, Velilla JM, Lobo A, Martín A, Paumard C, et al. Estudio de prevalencia de trastornos de la conducta alimentaria en adolescentes de Zaragoza. Revista de Psiquiatría Infanto Juvenil 1998;3:148-60.
36. Torres Y, Montoya I. Segundo estudio nacional de salud mental y consumo de sustancias psicoactivas. Santafé de Bogotá D.C.: Ministerio de Salud de Colombia; 1997.

37. Silva LC. Muestreo para la Investigación de ciencias de la salud. Madrid: Díaz de Santos; 1993.

38. Pólit D, Hungler B. Investigación científica en ciencias de la salud: Principios y métodos. Sexta edición. México: McGraw Hill Interamericana; 2000.

39. Hernández M, Garrido F, Salazar E. Sesgos. En: Hernández M. Epidemiología: diseño y análisis de estudios. México: Editorial Médica Panamericana; 2007. p. 253-68. 